CrossMark \&Click for updates

Cite this: Mol. BioSyst., 2017, 13,432

DOI: $10.1039 / \mathrm{c} 7 \mathrm{mb} 90005 \mathrm{~h}$

www.rsc.org/molecularbiosystems

\section{Correction: Elucidating time-dependent changes in the urinary metabolome of renal transplant patients by a combined ${ }^{1} \mathrm{H}$ NMR and GC-MS approach}

\author{
Kienana Muhrez, ${ }^{a}$ Lydie Nadal-Desbarats, ${ }^{\text {bc }}$ Jean-Michel Halimi, ${ }^{\text {ad }}$ Binta Dieme, $^{\text {b }}$ \\ Matthias Büchler, ${ }^{\text {ad }}$ Patrick Emond, ${ }^{\text {bc }}$ Hélène Blasco ${ }^{b}$ and Chantal Le Guellec $\dagger^{\star a}$
}

Correction for 'Elucidating time-dependent changes in the urinary metabolome of renal transplant patients by a combined ${ }^{1}$ H NMR and GC-MS approach' by Kienana Muhrez et al., Mol. BioSyst., 2015, 11, 2493-2510.

There was an error in the formatting of the author names in the original manuscript. The correct author names are indicated above.

The Royal Society of Chemistry apologises for these errors and any consequent inconvenience to authors and readers.

\footnotetext{
${ }^{a}$ Cellules dendritiques, immuno-intervention et greffes, EA4245, Université François Rabelais, Faculté de médecine, bâtiment Vialle, 10 Boulevard Tonnellé, 37032 Tours Cedex 1, France.E-mail: kienana.muhrez@etu.univ-tours.fr, halimi@med.univ-tours.fr, buchler@med.univ-tours.fr

${ }^{b}$ Equipe neurogénétique et neurométabolomique, INSERM U930, Université François Rabelais, Faculté de médecine, bâtiment Bretonneau, 10 Boulevard Tonnellé, 37044 Tours Cedex 9, France.E-mail: lydie.nadal@univ-tours.fr, bintdiem88@yahoo.fr, emond@univ-tours.fr, helene.blasco@univ-tours.fr 'Département d'Analyse Chimique Biologique et Médicale. PPF "Analyses des Systèmes Biologiques", Université François-Rabelais, Faculté de médecine, bâtiment Bretonneau, 10 Boulevard Tonnellé, 37032 Tours Cedex 1, France

${ }^{d}$ Service de néphrologie-immunologie clinique, CHU, hôpital Bretonneau, 2 bis boulevard Tonnellé, 37044 Tours, France

$\dagger$ Unité de Pharmacogénétique, Laboratoire de Biochimie et de Biologie Moléculaire, CHU, Hôpital Bretonneau, 2 bis boulevard Tonnellé - 37044 Tours Cedex, France. E-mail: chantal.barin-leguellec@univ-tours.fr; Fax: +33-02-47-47-86-13; Tel: +33-02-47-47-80-60.
} 